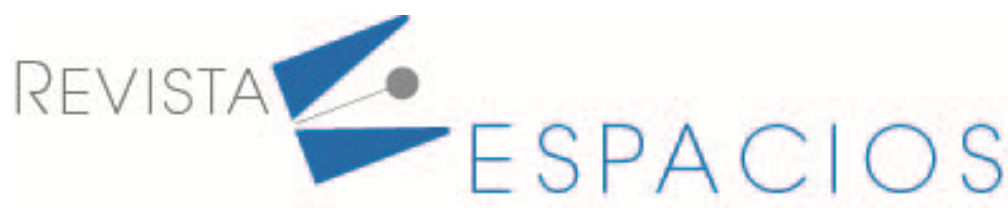

Vol. 41 (47) $2020 \cdot$ Art. 6

\title{
Revisión de los factores de emisión en las metodologías de huella de carbono en Colombia
}

\section{Review of emission factors in carbon footprint methodologies in Colombia}

RODRÍGUEZ, Juan P. ${ }^{1}$ RUIZ-OCHOA, Mauricio A. ${ }^{2}$

MENESES, Alexander ${ }^{3}$

\begin{abstract}
Resumen
Una revisión de los factores de emisión fue realizada en diversas metodologías asociadas a la cuantificación de la huella de carbono. Asimismo, se hizo un análisis de las fuentes de emisión que liberan los gases efecto invernadero (GEI), de las instalaciones (en un límite geográfico) e incidencias de los GEI según la incertidumbre (resultado de la cuantificación que caracteriza la dispersión de los valores).
\end{abstract}

Palabras clave: Huella de carbono, gases efecto invernadero, factores de emisión.

\begin{abstract}
A review of emission factors was carried out in various methodologies associated with the quantification of the carbon footprint. As well as, an analysis of emission sources that release greenhouse gases (GHGs) from the facilities (at a geographical limit) and GHG impacts according to the uncertainty (result of the quantification that characterizes the dispersion of the values).

Key words: Carbon footprint, greenhouse gases, emission factors.
\end{abstract}

\section{Introducción}

La humanidad confronta el problema de la contaminación ambiental a escala global asociada a la emisión de gases efecto invernadero (GEI) de origen antrópico, tanto los de bajo potencial de calentamiento global, pero más abundantes como el dióxido de carbono $\left(\mathrm{CO}_{2}\right)$ y el metano $\left(\mathrm{CH}_{4}\right)$, así como los de alto potencial de calentamiento, como el óxido nitroso $\left(\mathrm{N}_{2} \mathrm{O}\right)$, los $\mathrm{HFCs}$ y el $\mathrm{SF}_{6}$ (IPCC, 2007). A la fecha los resultados en materia de reducciones concertadas de emisiones GEI y por consiguiente en la regulación de la temperatura del planeta son preocupantes, dado que el objetivo de no permitir un aumento mayor a $2^{\circ} \mathrm{C}$ con respecto a la era preindustrial, no fue cumplido y no podría cumplirse de mantener los acuerdos revisados de Kyoto en el COP16, en materia de actividades de reducción, mitigación y control de GEI (IPCC, 2014).

\footnotetext{
${ }^{1}$ Ingeniero Sanitario y Ambiental. Magister en Ingeniería Ambiental. PhD. en Ingeniería. Profesor Asociado. Universidad Distrital Francisco José de Caldas. Director del grupo de investigación AQUAFORMAT. Correo electrónico: jprodriguezm@udistrital.edu.co. Dirección Postal: Carrera 5 Este No 15 - 82. Avenida Circunvalar Venado de Oro. Bogotá D.C. Colombia.

2 Ingeniero Ambiental. Magister en Ingeniería - Recursos Hidráulicos. Ph.D. en Ingeniería. Docente Investigador. Universidad Manuela Beltrán. Bucaramanga. Colombia.

${ }^{3}$ Ingeniero Químico. Magister en Ingeniería Química. Magíster en Química del Ambiente y la Energía. Ph.D en Ingeniería de Sistemas Energéticos. Grupo de Bioprocesos y Flujos Reactivos Universidad Nacional de Colombia, Sede Medellín.
} 
Esto quiere decir por ejemplo, que las metas en apariencia ambiciosas de Estados Unidos (Obama's Clean Power Plan) de reducir 26\% sus emisiones de GEl con respecto al inventario del año 2005 o de la Unión Europea de reducir 40\%, junto con el objetivo (no aceptado) que correspondería a China de reducir hasta $60 \%$ sus emisiones GEI, no sería suficientes, a pesar de que en conjunto estas regiones suman $~ 55 \%$ de las emisiones globales de

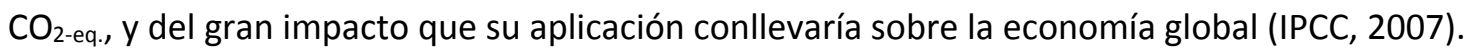

En efecto, aplicando los modelos de predicción de aumento de la temperatura planetaria y de acumulación de GEI del Panel Intergubernamental del Cambio Climático (IPCC por sus iniciales en inglés), la temperatura en un escenario optimista en el mejor de los casos subiría 2.7 a $3^{\circ} \mathrm{C}$, pero otros modelos estiman aumentos de más de $5^{\circ} \mathrm{C}$. Sin embargo, la primera parte del objetivo es decir el ajuste a los $2^{\circ} \mathrm{C}$ establecido en el COP2 1 (http://www.cop21.gouv.fr/es/), demandaría reducciones de GEI que de acuerdo a los expertos son difíciles de alcanzar y que en el mejor de los casos serían sostenibles solo hasta 2030. En general, los acuerdos del COP21 que propenden por una reducción más drástica de las emisiones de GEl, también buscan fortalecer las metodologías para su cuantificación y los mecanismos concertados de reducción de estos tipos de contaminantes, tales como el mecanismo de desarrollo limpio (MDL), la implementación conjunta (IC) o los mercados de carbono (IPCC, 2014).

Específicamente, el fortalecimiento de los protocolos de medición y verificación de las emisiones de GEl y sus concentraciones en la atmósfera, compromete la revisión permanente de los factores de emisión de los distintos GEI, y por consiguiente afecta su uso en el cálculo de otros indicadores ambientales, de uso cada vez más frecuente en la evaluación de impacto ambiental, como es el caso de las denominadas huellas de carbono (carbon footprint), en sus diferentes variantes. Estas incluyen desde la expresión básica de la masa (kg., ton.) de $\mathrm{CO}_{2 \text {-eq. }}$ emitida por una organización, proceso, sistema o actividad, por unidad de producto o periodo de tiempo, hasta su forma más estricta, que corresponde al cálculo del área de territorios productivos (cultivos, pastos, bosques, superficies de cuerpos de agua, etc.), requeridos para secuestrar una determinada masa de carbono emitida por una actividad u organización (http://www.footprintnetwork.org/our-work/climate-change/).

Por su parte, los factores de emisión de base que sean utilizados para estimar la huella de carbono, deben ser claramente entendidos, por cuanto este puede referirse estrictamente a las emisiones directas de $\mathrm{CO}_{2 \text {-eq }}$ producidas por la actividad o sistema o incluir emisiones indirectas conexas a la actividad, sistema u organización, fuera de sus fronteras físicas o tradicionales, en cuyo caso la huella de carbono responde a una frontera ampliada o de mayor alcance, la cual es característica del enfoque ACV o de Análisis de Ciclo de Vida (Wu et al., 2015), el cual le confiere a la huella calculada, el carácter de herramienta de gestión ambiental y de indicador de uso reconocido y extendido, dado que promueve la construcción de estrategias de mitigación y de compensación de los GEI en la cadena de suministro o ciclo de vida del sistema evaluado (Carballo et al., 2009; Espíndola \& Valderrama, 2012; CAEM, 2013; SDA, 2013; López et al., 2016), o para desarrollar estrategias de economía circular (Mohan et al., 2016a; Mohan et al., 2016b; D'Amato et al., 2017).

En efecto, la metodología de huella de carbono se considera un ACV simplificado o selectivo de indicador único (Wu et al., 2015; Ihobe, 2013), cuyo nicho principal se encuentra actualmente en el sector de la construcción sostenible (Solís-Guzmán et al., 2013; Martínez-Rocamora et al., 2017; Pham et al., 2018); la comparación de modos de producción agrícola (Page et al., 2012; Ramírez et al., 2015; Pérez-Neira et al., 2018) y como indicador de impacto urbano global (Pandey \& Venkataramana, 2014; Ramachandra et al., 2015; Singh et al., 2017), además de incursionar en el campo del estudio de hábitos de consumo individuales (Chakraborty \& Roy, 2013; Meza et al., 2013; Urge-Vorsatz et al., 2013) e institucionales, como es el caso de la iniciativa de cálculo de la huella ecológica de instituciones educativas (Aroonsrimorakot et al., 2013; Utaraskul, 2015; Robinson et al., 2018). 
En general, existen diversas metodologías que permiten elaborar inventarios de los GEl emitidos por distintas actividades antrópicas, pero su base son los factores de emisión (Ferrero et al., 2013; Solano-Quezada \& OrtízMalavassi, 2016), entendiéndose éstos, como la relación entre la cantidad de GEI emitido a la atmósfera y una unidad de producción o una unidad funcional (enfoque ACV). Estas relaciones son dinámicas y cambiantes en el tiempo, debido a razones metodológicas relacionadas con estudios que conceptualizan y recomiendan el ajuste nominal del factor de emisión de una determinada actividad o sector (Wu et al., 2015) o a razones prácticas, como por ejemplo cambios de tecnología que pueden modificar radicalmente el escenario de emisiones producidas o emitidas, un aspecto que permea la praxis de todas las metodologías basadas en ACV (Pehnt, 2006).

Con base en estas consideraciones generales, este artículo considera la revisión de algunos factores de emisión de uso frecuente, en las metodologías de huella de carbono y su adaptación al contexto colombiano. Así mismo se hace un análisis de las fuentes de emisión (unidad o proceso físico que libera GEI), instalaciones (en un límite geográfico) e incidencias de los GEI según la incertidumbre (resultado de la cuantificación que caracteriza la dispersión de los valores) y fenómenos climatológicos.

\section{Metodología}

Con base en las generalidades anteriores, este artículo considera la revisión de algunos factores de emisión de uso frecuente en las metodologías de huella de carbono, y su adaptación al contexto colombiano. Así mismo, se hace un análisis de las fuentes de emisión (unidad o proceso físico que libera GEI), instalaciones (en un límite geográfico), e incidencias de los GEI según la incertidumbre (resultado de la cuantificación que caracteriza la dispersión de los valores) y los fenómenos climatológicos.

\section{Resultados}

\subsection{Metodologías de huella de carbono}

Las metodologías de huella de carbono, presentan varias técnicas de cuantificación. En la revisión realizada se encontraron 15 ampliamente descritas: PAS 2050:2011 (Specification for the assessment of the life cycle greenhouse gas emissions of goods and services), Korea PCF (Corea), Carbon Footprint Program (Requirements and guidelines for quantification and communication), Carbon Index Casino (Francia), Greenext (Francia), Climate Certification System (Suecia), Climatop (Suiza), BP X30 - 323 (Francia), GHG Protocol (Product Life Cycle Accounting and Reporting Standard), ISO/TS 14067:2013, Greenhouse Gases, Ecoinvent 2.2, XM expertos, MC3 (Incluye emisiones directas e indirectas de los combustibles fósiles), IPCC 2012 GHG calcula los GEI provenientes de diferentes fuentes y sectores, e incluye una lista detallada de factores de emisión, y Balan Carbone (Domenech \& Arenales, 2008; Lemaignan \& Wilmotte, 2013; Espíndola \& Valderrama, 2016; Nevado et al., 2016; SolanoQuezada \& Ortíz-Malavassi, 2016). En el Cuadro 1 se presenta una comparación entre cinco de las técnicas de cálculo de huella de carbono.

Se encontró que estas técnicas difieren en la utilidad, en la contabilización de los GEl, en el uso internacional, y en la certificación/verificación (Cuadro 1). Sin embargo, es importante resaltar que, en el caso de los métodos del World Resources Institute (WRI), en la mayoría de ellos el $\mathrm{CH}_{4}$ y el $\mathrm{CO}_{2}$ biogénicos si hacen parte del cálculo, a diferencia de las otras metodologías que se apegan estrictamente al IPCC. 
Cuadro 1

Comparación de algunas técnicas de cálculo de huella de carbono.

\begin{tabular}{|c|c|c|c|c|c|}
\hline & $\begin{array}{c}\text { UNE-EN ISO } \\
14.067\end{array}$ & $\begin{array}{l}\text { GHG Procotol } \\
\text { Alcance } 1 \text { y } 2\end{array}$ & $\begin{array}{c}\text { GHG Procotol } \\
\text { Alcance } 3\end{array}$ & Bilan Carbone & PAS 2050:2011 \\
\hline $\begin{array}{l}\text { Organización } \\
\text { responsable }\end{array}$ & $\begin{array}{c}\text { Organización } \\
\text { Internacional de } \\
\text { Normalización }\end{array}$ & $\begin{array}{l}\text { World Business } \\
\text { Council for } \\
\text { Sustainable } \\
\text { Development World } \\
\text { Resources Institute }\end{array}$ & $\begin{array}{l}\text { World Business } \\
\text { Council for } \\
\text { Sustainable } \\
\text { Development } \\
\text { World Resources } \\
\text { Institute }\end{array}$ & ADEME & $\begin{array}{l}\text { British Standard } \\
\text { Institute }\end{array}$ \\
\hline Utilidad & $\begin{array}{l}\text { Inventario de } \\
\text { emisiones }\end{array}$ & $\begin{array}{l}\text { Inventario de } \\
\text { emisiones }\end{array}$ & Huella de carbono & $\begin{array}{l}\text { Huella de } \\
\text { carbono }\end{array}$ & $\begin{array}{c}\text { Huella de carbono y } \\
\text { compensación de } \\
\text { emisiones }\end{array}$ \\
\hline $\begin{array}{l}\text { Recomendaciones } \\
\text { para la reducción }\end{array}$ & Sí & No & No & Sí & Sí \\
\hline $\begin{array}{l}\text { Recomendaciones } \\
\text { para la } \\
\text { compensación }\end{array}$ & No & No & No & No & Sí \\
\hline $\begin{array}{l}\text { Contabilización de } \\
\text { las remociones de } \\
\text { GEI }\end{array}$ & Sí & No & No & No & No \\
\hline $\begin{array}{l}\text { Gases } \\
\text { considerados }\end{array}$ & Todos los GEI & $\begin{array}{c}6 \text { incluidos en el } \\
\text { Protocolo de Kioto } \\
\left(\mathrm{CO}_{2}, \mathrm{CH}_{4}, \mathrm{~N}_{2} \mathrm{O}, \mathrm{HFCs},\right. \\
\left.\text { PFCs, } \mathrm{SH}_{6}\right)\end{array}$ & $\begin{array}{c}6 \text { incluidos en el } \\
\text { Protocolo de Kioto } \\
\left(\mathrm{CO}_{2}, \mathrm{CH}_{4}, \mathrm{~N}_{2} \mathrm{O}\right. \\
\left.\mathrm{HFCs}, \mathrm{PFCs}, \mathrm{SH}_{6}\right)\end{array}$ & $\begin{array}{c}6 \text { incluidos en } \\
\text { el Protocolo de } \\
\text { Kioto }\left(\mathrm{CO}_{2}, \mathrm{CH}_{4},\right. \\
\mathrm{N}_{2} \mathrm{O}, \mathrm{HFCs}, \\
\left.\text { PFCs, } \mathrm{SH}_{6}\right)\end{array}$ & $\begin{array}{c}6 \text { incluidos en el } \\
\text { Protocolo de Kioto } \\
\left(\mathrm{CO}_{2}, \mathrm{CH}_{4}, \mathrm{~N}_{2} \mathrm{O}\right. \\
\left.\mathrm{HFCs}, \mathrm{PFCs}, \mathrm{SH}_{6}\right)\end{array}$ \\
\hline Escala & Organización & $\begin{array}{l}\text { Organización / } \\
\text { Producto / } \\
\text { Servicio }\end{array}$ & $\begin{array}{c}\text { Organización / } \\
\text { Producto / Servicio }\end{array}$ & $\begin{array}{l}\text { Organización / } \\
\text { Producto / } \\
\text { Servicio }\end{array}$ & $\begin{array}{l}\text { Organización / } \\
\text { Producto }\end{array}$ \\
\hline Alcance & $\begin{array}{c}\text { Directas + } \\
\text { Indirectas + } \\
\text { otras indirectas }\end{array}$ & $\begin{array}{l}\text { Directas + } \\
\text { Indirectas }\end{array}$ & $\begin{array}{c}\text { Directas + } \\
\text { Indirectas + otras } \\
\text { indirectas } \\
\end{array}$ & $\begin{array}{c}\text { Directas + } \\
\text { Indirectas+ } \\
\text { otras indirectas } \\
\end{array}$ & $\begin{array}{c}\text { Directas + } \\
\text { Indirectas+ otras } \\
\text { indirectas } \\
\end{array}$ \\
\hline Uso internacional & Sí & Sí & Sí & No & Sí \\
\hline $\begin{array}{l}\text { Posibilidad de } \\
\text { verificación por } \\
\text { un organismo } \\
\text { externo } \\
\text { independiente } \\
\end{array}$ & Sí & Sí & Sí & $\begin{array}{l}\text { No. La } \\
\text { verificación la } \\
\text { realiza ADEME }\end{array}$ & Sí \\
\hline & Sí & No & No & No & Sí \\
\hline $\begin{array}{l}\text { Certificación / } \\
\text { verificación }\end{array}$ & $\begin{array}{c}\text { Permite la } \\
\text { verificación de los } \\
\text { inventarios y } \\
\text { emisiones reducidas. } \\
\text { Certificación a nivel } \\
\text { de ISO. }\end{array}$ & $\begin{array}{l}\text { No es un estándar de } \\
\text { verificación (ofrece } \\
\text { guías para hacerlo } \\
\text { verificable). No } \\
\text { certifica las } \\
\text { verificaciones. }\end{array}$ & $\begin{array}{l}\text { No es un estándar } \\
\text { de verificación } \\
\text { (ofrece guías para } \\
\text { hacerlo } \\
\text { verificable). No } \\
\text { certifica las } \\
\text { verificaciones. }\end{array}$ & $\begin{array}{l}\text { No es un } \\
\text { estándar de } \\
\text { verificación. No } \\
\text { certifica las } \\
\text { verificaciones. }\end{array}$ & $\begin{array}{c}\text { Permite la } \\
\text { verificación de los } \\
\text { inventarios y } \\
\text { emisiones } \\
\text { reducidas. Certifica } \\
\text { la neutralidad } \\
\text { climática de la } \\
\text { empresa. }\end{array}$ \\
\hline
\end{tabular}

Fuente: Ihobe (2013)

Por otra parte, las estimaciones de las emisiones según la fuente se pueden establecer como (MINAM, 2010):

$$
\text { Emisión }_{\text {Fuente GEI }}=\text { Factor }_{\text {emisión }} * \text { Actividad, }
$$

donde la actividad, viene dada por la cantidad de combustible consumido (galones, $\mathrm{m}^{3}, \mathrm{kwh}$ ), emisiones por las actividades de transporte, aéreo, terrestre o generación de energía; el factor de emisión, el cual puede ser 
calculado de formas diferentes (cálculo del margen combinado, margen de operación y el margen de construcción), y los valores pueden ser relacionados por la literatura de manera amplia, obtenidos de premisas que aportan fiabilidad, precisión, exactitud y representatividad. De este modo, los factores de emisión, para la estimación de la huella de carbono, considerados como datos de información secundaria pueden ser diferentes (Andrade et al., 2014; SDA, 2013; de Toro et al., 2014; Hermosilla, 2014; Herrera 2014; Muñiz et al., 2016; Roig, 2016; Gavilán \& Reinoso, 2017), tal como se observa en el Cuadro 2.

Cuadro 2

Comparación de algunas técnicas de cálculo de huella de carbono.

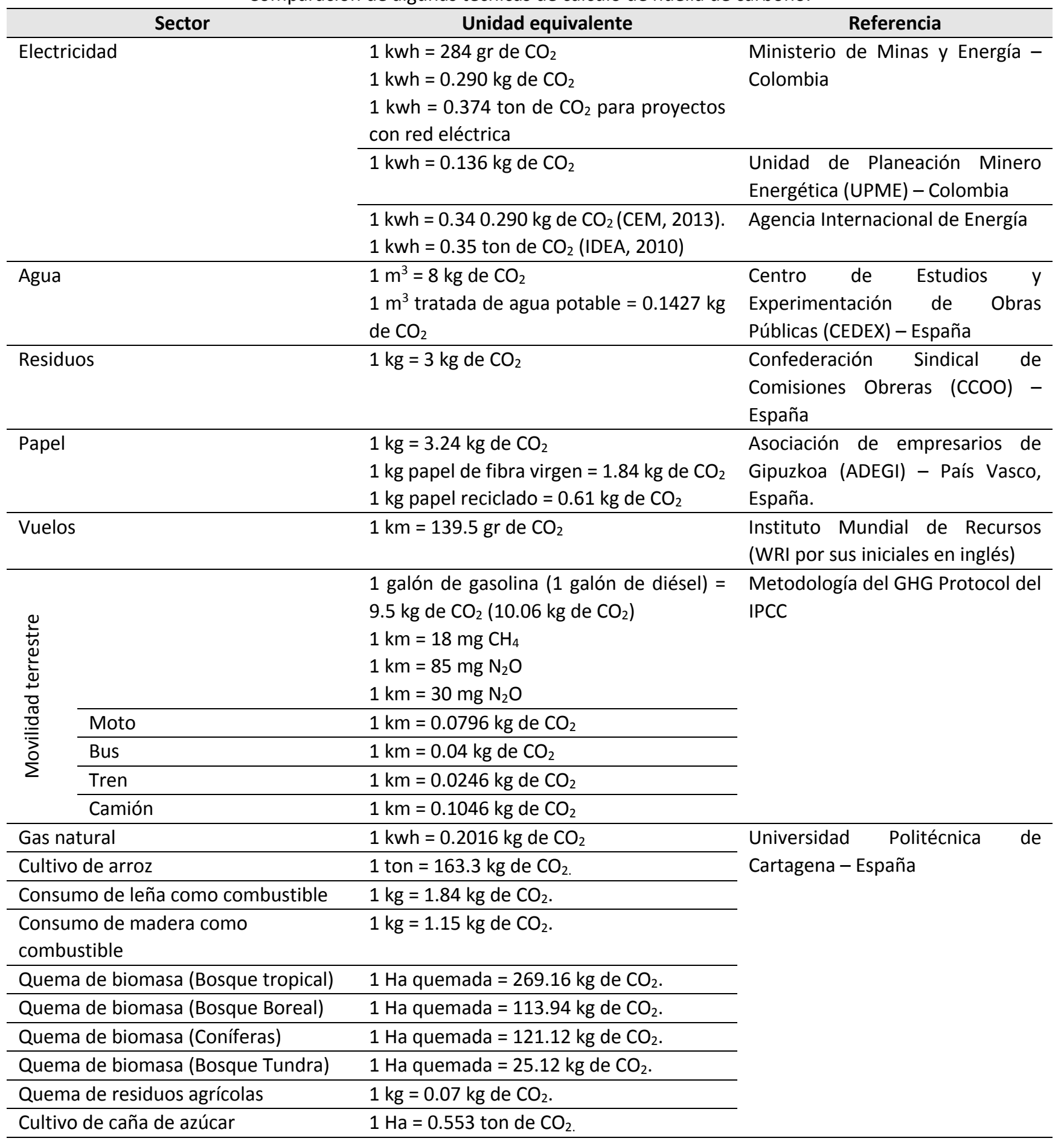




\begin{tabular}{|c|c|c|}
\hline Sector & Unidad equivalente & Referencia \\
\hline Construcción & $\begin{array}{l}1 \mathrm{~m}^{2} \text { de construcción o construido }=405 \\
\mathrm{~kg} \mathrm{de} \mathrm{CO}_{2}\left(520 \mathrm{~kg} \text { de } \mathrm{CO}_{2}\right)\end{array}$ & \\
\hline Vivienda & 1 habitante (vivienda) $=0.274$ ton de $\mathrm{CO}_{2}$ & \\
\hline $\begin{array}{l}\text { Tratamiento de aguas residuales } \\
\text { domésticas }\end{array}$ & $1 \mathrm{Hab}=73 \mathrm{~kg}$ de $\mathrm{CO}_{2}$ & \\
\hline $\begin{array}{l}\text { Tratamiento de aguas residuales } \\
\text { industrial }\end{array}$ & $1 \mathrm{~kg} \mathrm{DQO}=4.73 \mathrm{~kg}$ de $\mathrm{CO}_{2}$. & \\
\hline Tratamiento de lodos & $1 \mathrm{~kg} \mathrm{DQO}=5.63 \mathrm{~kg} \mathrm{de} \mathrm{CO}_{2}$. & \\
\hline Disposición de residuos sólidos & $1 \mathrm{~kg}$ residuos sólidos $=5.13 \mathrm{~kg}$ de $\mathrm{CO}_{2}$. & \\
\hline & $1 \mathrm{~kg}=3.35 \mathrm{~kg}$ de $\mathrm{CO}_{2}$ & \\
\hline Insecticida & $1 \mathrm{~kg}=25.13 \mathrm{~kg}$ de $\mathrm{CO}_{2}$ & \\
\hline$\sqcup \stackrel{N}{L}$ Fungicida & $1 \mathrm{~kg}=6.009 \mathrm{~kg}$ de $\mathrm{CO}_{2}$ & \\
\hline $\begin{array}{l}\text { Extracción de carbón (mina } \\
\text { subterránea) }\end{array}$ & 1 ton $=312.39 \mathrm{~kg} \mathrm{de} \mathrm{CO}_{2}$ & \\
\hline $\begin{array}{l}\text { Extracción de carbón (mina cielo } \\
\text { abierto) }\end{array}$ & 1 ton $=20.94 \mathrm{~kg}$ de $\mathrm{CO}_{2}$ & \\
\hline Producción de coque & 1 ton $=12.5 \mathrm{~kg}$ de $\mathrm{CO}_{2}$ & \\
\hline Producción de cemento & 1 ton $=507.1 \mathrm{~kg}$ de $\mathrm{CO}_{2}$ & \\
\hline Producción de cal viva & 1 ton $=790 \mathrm{~kg}$ de $\mathrm{CO}_{2}$ & \\
\hline Producción de cal dolomita & 1 ton $=910 \mathrm{~kg}$ de $\mathrm{CO}_{2}$ & \\
\hline Producción de amoniaco & 1 ton $=1500 \mathrm{~kg}$ de $\mathrm{CO}_{2}$ & \\
\hline
\end{tabular}

Teniendo en cuenta lo anterior, la incertidumbre considerada como la "cantidad que permite asignar un cierto grado de confianza al resultado de una medida y determinar la probabilidad de que el resultado de la medida esté más o menos cerca del valor buscado" (Alòs \& Nualart, 2003), deber analizarse desde las perspectivas de las relaciones matemáticas, ecuaciones o modelos utilizados para caracterizar los vínculos entre parámetros y procesos de emisión (WRI, 2016).

Además, si se tiene la incertidumbre de los parámetros, la cual se refiere a la incertidumbre asociada a la cuantificación de los parámetros utilizados como insumos (datos de actividad o factores de emisión) en los modelos de estimación (WRI, 2016). Por lo tanto, la incertidumbre se calcula y varía de acuerdo con la cantidad de datos disponibles. Si solamente se cuenta con un dato, la incertidumbre de la variable será estimada y corresponderá al dato propuesto por el panel de expertos del IPCC, descrito en el documento: "Orientación del IPCC sobre las buenas prácticas y la gestión de la incertidumbre en los inventarios nacionales de gases de efecto invernadero". Pero si por el contrario, se cuenta con dos o más datos para el cálculo de las emisiones de GEI, el resultado de la incertidumbre de la variable será calculado según la metodología propuesta por el IPCC y adoptada por el GHG Protocol, descrita en el documento: "Guía corta para el cálculo de la medición y estimación de la incertidumbre para emisiones de GHG".

\subsection{Metodologías para el cálculo de huella de carbono en Colombia}

En Colombia, la Huella de Carbono ha ganado notoriedad desde la COP21. Se han generado diferentes metodologías desde el sector público y privado. El Ministerio de Ambiente y Desarrollo Sostenible, la mayor autoridad ambiental en Colombia ha desarrollado la Calculadora 2050; basado en la metodología PAS2050. Esta es definida como una herramienta de planeación y toma de decisiones que proyecta diferentes escenarios de reducción de las emisiones de $\mathrm{GEI}$, teniendo en cuenta los diferentes sectores de la economía. 
Previo al diseño de cálculos se analizaron las necesidades de Colombia para adaptar la herramienta del Reino Unido y se definió conjuntamente con la Estrategia Colombiana de Desarrollo Bajo en Carbono (ECDBC), Ios sectores, componentes y medidas de mitigación de emisiones a ser incluidos en la Calculadora de Carbono 2050. Por su parte, la Secretaria de Ambiente Distrital (SDA, 2013), autoridad en temas ambientales en la capital de la república, Bogotá, aborda la mitigación del cambio climático desde el cálculo de la huella de carbono de empresas en la ciudad. Para ello utiliza una herramienta diseñado sobre la metodología del GHG Protocol, el cual puede incluir emisiones biogénicas de $\mathrm{CH} 4$ y $\mathrm{CO} 2$, lo cual genera un distanciamiento con IPCC y en términos de negociaciones de línea base para muchos sectores esto es penalizante, como por ejemplo en las hidroeléctricas. A su vez, el Instituto Colombiano de Normas Técnicas (ICONTEC), ha generado estándares que están en concordancia con los lineamientos internacionales, enmarcados en la NTC 5947: "Especificación para el análisis de emisiones y remociones de Gases Efecto Invernadero durante el ciclo de vida de bienes y servicios", la cual se basa en las metodologías del PAS 2050 para el Análisis de Ciclo de Vida (ACV), la ISO14044 y los factores de emisión del IPCC 2007.

De igual modo, la Corporación Autónoma Regional de Cundinamarca (CAR), dentro de las Estrategias del Plan de Gestión Ambiental Regional (PGAR) 2012-2023, introdujo el Cambio Climático (CC) como unas de sus prioridades. Así, en el 2013, la CAR, en compañía de la Corporación Ambiental Empresarial (CAEM) y de la Cámara de Comercio Bogotá (CCB) elaboró La Guía Metodológica para el Cálculo de la Huella De Carbono a Nivel Sectorial, la cual buscaba identificar oportunidades a nivel sectorial para reducir las emisiones de GEl. En este sentido, Mutis (2016) identificó el enfoque metodológico, la pertinencia y la aplicabilidad en la reducción de la huella de carbono de dicha guía, y encontró que, la guía metodológica en la parte técnica es muy completa, sin embargo, la guía no contiene aspectos que permitan al usuario una vez calculada la huella de carbono, interpretar y disminuir sus emisiones de gases de efecto invernadero.

Uno de los principales problemas detectados en las metodologías, es la falta de cercanía que guardan los factores de emisión con las condiciones del país, como la composición fisicoquímica del combustible; por lo que el grado de incertidumbre (Nivel de confianza) de la Huella de Carbono tiende al aumento. Basado en lo anterior, la Unidad de Planeación Minero Energética (UPME) en 2003, en conjunto con la Academia Colombiana de Ciencias exactas, Físicas y Naturales (ACCEFYN) realizó el levantamiento de factores de emisión para 68 combustibles. Los cálculos se fundamentaron en las bases teóricas suministradas por la estequiometria de cada uno de los combustibles, establecida a partir de su composición elemental, obteniéndose los factores de emisión para los combustibles líquidos, sólidos y gaseosos (Cuadro 3)

Cuadro 3

Comparación de algunas técnicas

de cálculo de huella de carbono.

\begin{tabular}{|c|c|c|c|c|}
\hline \multirow{2}{*}{$\begin{array}{c}\text { Tipo de } \\
\text { Combustible }\end{array}$} & \multirow{2}{*}{ Combustible } & \multicolumn{2}{|c|}{ Factor de Emisión } & \multirow{2}{*}{ Referencia } \\
\hline & & Cantidad & Unidad & \\
\hline \multirow{11}{*}{ Líquido } & ACPM & 10.15 & $\mathrm{~kg} \mathrm{CO}_{2 \mathrm{e}} / \mathrm{gal}$ & CAR et al. (2013), Herrera (2014) \\
\hline & Combustóleo & 11.76 & $\mathrm{~kg} \mathrm{CO}_{2 \mathrm{e}} / \mathrm{gal}$ & CAR et al. (2013), Herrera (2014) \\
\hline & Cruda de castilla & 11.72 & $\mathrm{~kg} \mathrm{CO}_{2 \mathrm{e}} / \mathrm{gal}$ & CAR et al. (2013), Herrera (2014) \\
\hline & Diésel genérico & 10.15 & $\mathrm{~kg} \mathrm{CO}_{2 \mathrm{e}} / \mathrm{gal}$ & CAR et al. (2013), Herrera (2014) \\
\hline & Diésel genérico & 2.69 & $\mathrm{~kg} \mathrm{CO} 2 \mathrm{e} / \mathrm{L}$ & MAGRAMA (2009) \\
\hline & Diésel genérico & 2.6502 & $\mathrm{~kg} \mathrm{CO} 2 \mathrm{e} / \mathrm{L}$ & CAR et al. (2013) \\
\hline & Gasolina genérico & 8.15 & $\mathrm{~kg} \mathrm{CO} 2 \mathrm{e} / \mathrm{gal}$ & CAR et al. (2013), Herrera (2014) \\
\hline & Gasolina genérico & 2.3018 & $\mathrm{~kg} \mathrm{CO} 2 \mathrm{e} / \mathrm{L}$ & CAR et al. (2013) \\
\hline & Gasolina genérico & 2.196 & $\mathrm{~kg} \mathrm{CO}_{2 \mathrm{e}} / \mathrm{L}$ & MAGRAMA (2009) \\
\hline & Gasolina genérico & 2.61 & $\mathrm{~kg} \mathrm{CO} 2 \mathrm{e} / \mathrm{L}$ & OCCC (2013) \\
\hline & Gasolina genérico & 2.196 & $\mathrm{~kg} \mathrm{CO}{ }_{2 \mathrm{e}} / \mathrm{L}$ & Nevado et al. (2016) \\
\hline
\end{tabular}




\begin{tabular}{|c|c|c|c|c|}
\hline \multirow{2}{*}{$\begin{array}{c}\text { Tipo de } \\
\text { Combustible }\end{array}$} & \multirow{2}{*}{ Combustible } & \multicolumn{2}{|c|}{ Factor de Emisión } & \multirow{2}{*}{ Referencia } \\
\hline & & Cantidad & Unidad & \\
\hline & Kersosene col & 9.71 & $\mathrm{~kg} \mathrm{CO}{ }_{2 \mathrm{e}} / \mathrm{gal}$ & CAR et al. (2013), Herrera (2014) \\
\hline & Kersosene genérico & 9.72 & $\mathrm{~kg} \mathrm{CO}_{2 \mathrm{e}} / \mathrm{gal}$ & CAR et al. (2013), Herrera (2014) \\
\hline & Oil crude & 11.54 & $\mathrm{~kg} \mathrm{CO} 2 \mathrm{e} / \mathrm{gal}$ & CAR et al. (2013), Herrera (2014) \\
\hline & Biodiesel genérico & 9.44 & $\mathrm{~kg} \mathrm{CO}_{2 \mathrm{e}} / \mathrm{gal}$ & CAR et al. (2013), Herrera (2014) \\
\hline & Biogasolina genérica & 7.17 & $\mathrm{~kg} \mathrm{CO}_{2 \mathrm{e}} / \mathrm{gal}$ & CAR et al. (2013), Herrera (2014) \\
\hline \multirow{5}{*}{ Sólido } & Bagazo & 1.68 & $\mathrm{~kg} \mathrm{CO} 2 \mathrm{e} / \mathrm{kg}$ & CAR et al. (2013), Herrera (2014) \\
\hline & Carbón genérico & 2.45 & $\mathrm{~kg} \mathrm{CO}_{2 \mathrm{e}} / \mathrm{kg}$ & CAR et al. (2013), Herrera (2014) \\
\hline & Fibra palma de aceite & 1.93 & $\mathrm{~kg} \mathrm{CO} 2 \mathrm{e} / \mathrm{kg}$ & CAR et al. (2013), Herrera (2014) \\
\hline & Leña & 1.84 & $\mathrm{~kg} \mathrm{CO} 2 \mathrm{e} / \mathrm{kg}$ & CAR et al. (2013), Herrera (2014) \\
\hline & Madera genérico & 1.15 & $\mathrm{~kg} \mathrm{CO} 2 \mathrm{e} / \mathrm{kg}$ & CAR et al. (2013), Herrera (2014) \\
\hline \multirow{12}{*}{ Gaseoso } & Biogás central & 1.97 & $\mathrm{~kg} \mathrm{CO} 2 \mathrm{e} / \mathrm{Nm}^{3}$ & CAR et al. (2013), Herrera (2014)) \\
\hline & Coke gas D & 0.77 & $\mathrm{~kg} \mathrm{CO} 2 \mathrm{e} / \mathrm{Nm}^{3}$ & CAR et al. (2013), Herrera (2014) \\
\hline & Gas domiciliario & 1.86 & $\mathrm{~kg} \mathrm{CO} 2 \mathrm{e} / \mathrm{Nm}^{3}$ & CAR et al. (2013), Herrera (2014) \\
\hline & Gas líquido D & 7.11 & $\mathrm{~kg} \mathrm{CO} 2 \mathrm{e} / \mathrm{Nm}^{3}$ & CAR et al. (2013), Herrera (2014) \\
\hline & Gas natural genérico & 56 & ton $\mathrm{CO}_{2 \mathrm{e}} / \mathrm{TJ}$ & CAR et al. (2013) \\
\hline & Gas natural genérico & 0.29 & $\mathrm{~kg} \mathrm{CO} 2 \mathrm{e} / \mathrm{kwh}$ & de Toro et al. (2014) \\
\hline & Gas natural genérico & 1.86 & $\mathrm{~kg} \mathrm{CO} 2 \mathrm{e} / \mathrm{Nm}^{3}$ & CAR et al. (2013), Herrera (2014) \\
\hline & LNG genérico & 1.86 & $\mathrm{~kg} \mathrm{CO} 2 \mathrm{e} / \mathrm{Nm}^{3}$ & CAR et al. (2013), Herrera (2014) \\
\hline & LPG genérico & 7.11 & $\mathrm{~kg} \mathrm{CO} 2 \mathrm{e} / \mathrm{Nm}^{3}$ & CAR et al. (2013), Herrera (2014) \\
\hline & LPG propano & 8.21 & $\mathrm{~kg} \mathrm{CO} 2 \mathrm{e} / \mathrm{Nm}^{3}$ & CAR et al. (2013), Herrera (2014) \\
\hline & GLP & 1.656 & $\mathrm{~kg} \mathrm{CO} 2 \mathrm{e} / \mathrm{L}$ & Nevado et al. (2016) \\
\hline & Oil Gas & 2.68 & $\mathrm{~kg} \mathrm{CO} 2 \mathrm{e} / \mathrm{Nm}^{3}$ & CAR et al. (2013), Herrera (2014) \\
\hline
\end{tabular}

Fuente: Autores

Dentro de las iniciativas privadas se encuentra la desarrollada por Ecologic SAS, que bajo la marca CO2CERO ${ }^{\circledR}$, comercializa los servicios de cálculo y compensación de huella de carbono. Para esto ha utilizado como base la metodología del GHG Protocol y complementada con los factores de emisión del IPCC. Para darle mayor validez utiliza factores nacionales de emisión de combustibles y vehículos. Para la medición de las emisiones asociadas al consumo energético, se actualiza la base de datos a diario generando una mayor confianza en el cálculo.

\section{Conclusiones}

La metodología de huella de carbono presenta diferencias notables (según estudios expuestos) en la conceptualización, significancia del indicador, contenido y diversidad en los métodos de cálculo, lo que considera el aumento de incertidumbre, la complejidad de representar las emisiones de gases no relacionados con el carbono (toneladas equivalente de $\mathrm{CO}_{2}$ ), y la alta variabilidad de los valores nominales de los factores de emisión, que hacen a esta metodología, una restringida medida de indicador de representar las emisiones de todos o parte de los gases efecto invernadero según el alcance de medición. Adicional a lo anterior, existe una gran divergencia metodológica que inhiben procesos de implementación debido a la variedad de los procedimientos y métodos para cuantificar las emisiones, especialmente en condiciones de la pequeña y mediana empresa, y la limitada capacidad de gestión de la información clara, precisa y coherente.

\section{Referencias bibliográficas}

Alòs, E. \& Nualart, D. (2003). Stochastic integration with respect to the fractional Brownian motion. Stochastic and Stochastic Reports, 75(3), 129-152. 
Andrade, H.; Campo, O. \& Segura, M. (2014). Huella de carbono del sistema de producción de arroz (Oryza sativa) en el municipio de Campoalegre, Huila, Colombia. Revista CORPOVA Ciencia Tecnología Agropecuaria, 15(1), 25-31.

Aroonsrimorakot, S.; Yuwaree, Ch.; Arunlertaree, Ch.; Hutajareorn, R. \& Buadit, T. (2013). Carbon Footprint of Faculty of Environment and Resource Studies, Mahidol University, Salaya Campus, Thailand. APCBEE Procedia, 5, 175-180. DOI: https://doi.org/10.1016/j.apcbee.2013.05.031.

Corporación Autónoma Regional de Cundinamarca (CAR), Corporación Ambiental Empresarial (CAEM), \& Cámara de Comercio Bogotá (CCB). (2013). Guía metodológica para el cálculo de la huella de carbono corporativa a nivel sectorial, Bogotá: Corporativa ambiental empresarial. Cámara de comercio de Bogotá.

Chakraborty, D. \& Roy, J. (2013). Energy and carbon footprint: numbers matter in low energy and low carbon choices. Current Opinion in Environmental Sustainability, 5(2), 237-243. DOI: https://doi.org/10.1016/j.cosust.2013.04.001.

Carballo, A.; García-Negro, M. \& Doménech, J.L. (2009). El MC3 una alternativa metodológica para estimar la huella corporativa de carbono (HCC). Revista Desarrollo Local Sostenible, 2(5), 1-16.

de Toro, A.; Gomera, A.; Aguilar, J.E.; Guijarro, C.; Antúnez, M. \& Vaquero, M. (2014). La Huella de Carbono de la Universidad de Córdoba. 2013, Córdoba, España: Universidad de Córdoba.

Domenech, J. \& Arenales, M. (2008). La huella ecológica de las empresas: 4 años de seguimiento en el puerto de Gijón. OIDLES, 1(4), 1-23.

D'Amato, D.; Droste, N.; Allen, B.; Kettunen, M.; Lähtinen, K.; Korhonen, J.; Leskinen, P.; Matthies, B.D. \& Toppinen, A. (2017). Green, circular, bio economy: A comparative analysis of sustainability avenues. Journal of Cleaner Production, 168, 716-734. DOI: https://doi.org/10.1016/j.jclepro.2017.09.053.

Espíndola, C., \& Valderrama, J. (2012). Huella del Carbono. Parte 1: Conceptos, Métodos de Estimación y Complejidades Metodológicas. Información Tecnológica, 23(1), 163-176. DOI: http://dx.doi.org/10.4067/S0718-07642012000100017.

Espíndola, C. \& Valderrama, J. (2016). AbaniCO2: Un Método Simple y Efectivo para la Toma de Decisiones sobre la Adopción de la Huella del Carbono en la Gestión Sustentable de Emisiones en las Empresas. Información Tecnológica, 27(3), 35-52. DOI: http://dx.doi.org/10.4067/S0718-07642016000300005.

Ferrero, R.; Gareis, M.C. \& Zulaica, L. (2013). Aportes para la estimación de la huella de carbono en los grandes asentamientos urbanos de Argentina. Revista Colombiana de Geografía, 22(2), 87-106. DOI: https://doi.org/10.15446/rcdg.v22n2.32770.

Gavilán, E. \& Reinoso, M. (2017). Estimación cuantitativa de la huella del carbono en el cultivo de la caña de azúcar en Villa Clara. Revista Centro Agrícola, 44(1), 71-79.

Hermosilla, A. (2014). Huella de Carbono en la Universidad Politécnica de Cartagena: En Busca de la Ecoeficiencia. Trabajo fin de Máster. Máster en Ingeniería Ambiental y de Procesos Sostenibles Universidad Politécnica de Cartagena (UPCT), Cartagena, España.

Herrera, H.H. (2014). Factores de emisión del S.I.N. Sistema Interconectado Nacional Colombia 2013. Bogotá, Colombia: Ministerio de Minas y energía. Bogotá.

Ihobe S.A. (2013). 7 Metodologías para el cálculo de emisiones de gases de efecto invernadero, Bilbao: Ihobe, Sociedad Pública de Gestión Ambiental.

Intergovernmental Panel on Climate Change (IPCC). (2007). Climate Change 2007: The Physical Science Basis. Contribution of Working Group I to the Fourth Assessment Report of the Intergovernmental Panel on 
Climate Change [Solomon, S.; Qin, D.; Manning, M.; Chen, Z.; Marquis, M.; Averyt, K.B.; Tignor, M. \& Miller, H.L. (eds.)]. Cambridge University Press, Cambridge, United Kingdom and New York, NY, USA.

IPCC. (2014). Climate Change 2014: Synthesis Report. Summary for policymakers. IPCC. USA: Intergovernmental Panel on Climate Change. NY, USA.

Meza, M.J.F.; Roy, J.; Urge-Vorsatz, D. \& Kahn Ribeiro, S. (2013). Energy systems in the context of sustainable development. Current Opinion in Environmental Sustainability, 5(2), 136-140. DOI: https://doi.org/10.1016/j.cosust.2013.05.001.

Lemaignan, B. \& Wilmotte, J.Y. (marzo, 2013). How to use the tool. Obtenido de http://www.geotechnicalcarboncalculator.com/es/.

López, B.; Rasero, P.; González, J. \& Álvarez-Ossorio, J. (2016). Inventario de emisiones de gases de efecto invernadero en el ciclo urbano del agua 2014. TECNOAQUA, 17, 96-101.

Martínez-Rocamora, A.; Solís-Guzmán, J. \& Marrero, M. (2017). Ecological footprint of the use and maintenance phase of buildings: Maintenance tasks and final results. Energy and Buildings, 155, 339-351. DOI: https://doi.org/10.1016/j.enbuild.2017.09.038.

Ministerio de Agricultura, Alimentación y Medio Ambiente (MAGRAMA). (2009). Inventarios Nacionales de Emisiones a la Atmósfera 1990-2012. Volumen 2: Análisis por Actividades SNAP. Capítulo 4. Madrid, España: Ministerio de Agricultura, Alimentación y Medio Ambiente.

Ministerio del Ambiente (MINAM). (2010). Huella de carbono del Ministerio del Ambiente (MINAN). Informe final. Lima, Perú: Ministerio de Ambiente.

Mohan, S.V.; Modestra, J.A.; Amulya, K.; Butti, S.K. \& Velvizhi, G. (2016a). A circular bioeconomy with biobased products from $\mathrm{CO}_{2}$ sequestration. Trends in Biotechnology, 34(6), 506-519. DOI: https://doi.org/10.1016/j.tibtech.2016.02.012.

Mohan, S.V.; Nikhil, G.N.; Chiranjeevi, P.; Nagendranatha Reddy, C.; Rohit, M.V.; Naresh Kumar, A. \& Sarkar, O. (2016b). Waste biorefinery models towards sustainable circular bioeconomy: Critical review and future perspectives. Bioresource Technology, 215, 2-12. DOI: https://doi.org/10.1016/j.biortech.2016.03.130.

Muñiz, I.; Rojas, C.; Busuldu, C.; García, A.; Filipe, M. \& Quintana, M. (2016). El impacto de la forma y estructura espacial urbana sobre las emisiones de $\mathrm{CO}_{2}$ en Concepción (Chile). ¿Es compatible una baja densidad residencial con un bajo nivel de emisiones?. Barcelona, España: Universidad de Barcelona. Departamento de economía aplicada.

Mutis, K. (2016). Análisis de la Guía Metodológica para el Cálculo de la Huella de Carbono Corporativa a Nivel Sectorial de la Corporación Autónoma Regional de Cundinamarca. Bogotá: Universidad Distrital Francisco José de Caldas. Especialización en Educación y Gestión Ambiental.

Nevado, S.; Gomis, A.; Mena, E. \& Castro, M. (2016). Cálculo de la huella de carbono del ciclo urbano del agua. La experiencia de EMUASA. Revista TECNOAQUA, 19, 58-65.

Oficina Catalana del Cambio Climático (OCCC). (2013). Guía Práctica para el Cálculo de Emisiones de Gases de Efecto Invernadero (GEI). Cataluña, España. 82 p.

Page, G.; Ridoutt, B. \& Bellotti, B. (2012). Carbon and water footprint tradeoffs in fresh tomato production. Journal of Cleaner Production, 32, 219-226. DOI: https://doi.org/10.1016/j.jclepro.2012.03.036.

Pandey, A. \& Venkataramana, Ch. (2014). Estimating emissions from the Indian transport sector with on-road fleet composition and traffic volume. Atmospheric Environment, 98, 123-133. DOI: https://doi.org/10.1016/j.atmosenv.2014.08.039. 
Pehnt, M. (2006). Dynamic life cycle assessment (LCA) of renewable energy technologies. Renewable Energy, 31(1), 55-71. DOI: https://doi.org/10.1016/j.renene.2005.03.002.

Pérez-Neira, D.; Soler-Montiel, M.; Delgado, M. \& Reigada, A. (2018). Energy use and carbon footprint of the tomato production in heated multi-tunnel greenhouses in Almeria within an exporting agri-food system context. Science of The Total Environment, 628-629, 1627-1636. DOI:

https://doi.org/10.1016/j.scitotenv.2018.02.127.

Pham, L.; Palaneeswaran, E. \& Stewart, R. (2018). Knowing maintenance vulnerabilities to enhance building resilience. Procedia Engineering, 212, 1273-1278. DOI: https://doi.org/10.1016/j.proeng.2018.01.164.

Ramachandra, T.V.; Aithal, B.H. \& Sreejith, K. (2015). GHG footprint of major cities in India. Renewable and Sustainable Energy Reviews, 44, 473-495. DOI: https://doi.org/10.1016/j.rser.2014.12.036.

Ramírez, T.; Meas, Y.; Dannehl, D.; Schuch, I.; Miranda, L.; Rocksch, T. \& Schmidt, U. (2015). Water and carbon footprint improvement for dried tomato value chain. Journal of Cleaner Production, 104, 98-108. DOI: https://doi.org/10.1016/j.jclepro.2015.05.007.

Robinson, O.J.; Tewkesbury, A.; Kemp, S. \& Williams, I.D. (2018). Towards a universal carbon footprint standard: A case study of carbon management at universities. Journal of Cleaner Production, 172, 44354455. DOI: https://doi.org/10.1016/j.jclepro.2017.02.147.

Roig, M. (2016). Determinación de la Huella de Carbono de productos agrícolas en una finca de agricultura ecológica. Valencia, España: Universidad Politécnica de Valencia. 56 p. DOI: http://hdl.handle.net/10251/76521.

Secretaría Distrital de Ambiente (SDA). (2013). Guía para la elaboración del informe de huella de carbono corporativa en entidades públicas del distrito capital. Bogotá: Subdirección de políticas y planes ambientales. Secretaria distrital de ambiente. 20 p.

Singh, R.; Sharma, Ch. \& Agrawal, M. (2017). Emission inventory of trace gases from road transport in India. Transportation Research Part D: Transport and Environment, 52(Part A), 64-72. DOI: https://doi.org/10.1016/j.trd.2017.02.011.

Solano-Quezada, S. \& Ortiz-Malavassi, E. (2016). Metodología de medición de la huella de carbono para edificaciones en Costa Rica y su aplicación el módulo habitacional Tropika. Tecnología en Marcha, 29(3), 73-84.

Solís-Guzmán, J.; Marrero, M. \& Ramírez-de-Arellano, A. (2013). A. Methodology for determining the ecological footprint of the construction of residential buildings in Andalusia (Spain). Ecological Indicators, 25, 239249. DOI: https://doi.org/10.1016/j.ecolind.2012.10.008.

Urge-Vorsatz, D.; Petrichenko, K.; Staniec, M. \& Eom, J. (2013). Energy use in buildings in a long-term perspective. Current Opinion in Environmental Sustainability, 5(2), 141-151. DOI: https://doi.org/10.1016/j.cosust.2013.05.004.

Utaraskul, T. (2015). Carbon Footprint of Environmental Science Students in Suan Sunandha Rajabhat University, Thailand. Procedia - Social and Behavioral Sciences, 197, 1156-1160. DOI: https://doi.org/10.1016/j.sbspro.2015.07.371.

World Resources Institute (WRI). (enero, 2016). A Corporate Accounting and Reporting Standard. Obtenido de http://pdf.wri.org/ghg_protocol_2004.pdf. 116 p.

Wu, P.; Xia, B. \& Wang, X. (2015). The contribution of ISO 14067 to the evolution of global greenhouse gas standards-A review. Renewable and Sustainable Energy Reviews, 47, 142-50. DOI: https://doi.org/10.1016/j.rser.2015.02.055.

Esta obra está bajo una Licencia Creative Commons Attribución-NoCommercial 4.0 International

(c) BY-NC 